\section{Wie sicher ist die ambulante direkte Laryngoskopie?}

\author{
Immer öfter werden direkte Laryngoskopien bei ambulanten Patienten \\ vorgenommen. Wie häufig es dabei zu Komplikationen kommt, wurde nun \\ in einer US-Studie untersucht.
}

\begin{abstract}
HO-Ärzte der Universität San Diego
haben dazu die Krankenakten von 7.743 erwachsenen Patienten angesehen, die sich 2010 oder 2011 ambulant einer direkten Laryngoskopie mit oder ohne Biopsie unterzogen hatten. In den ersten sieben Tagen nach der Intervention hatten sich bei weniger als $0,3 \%$ der Patienten schwerwiegende Atemwegskomplikationen eingestellt. Die Studienautoren um Ryan K. Orosco attestieren dem ambulanten Einsatz daher „ein hochakzeptables Sicherheitsprofil“. Sie betonen allerdings die Notwendigkeit einer sorgfältigen Patientenauswahl.

Die in der Studie berücksichtigten $\mathrm{Pa}$ tienten waren im Mittel 60 Jahre alt und überwiegend männlich. Die häufigsten Diagnosen waren Polypen und andere Erkrankungen der Stimmlippen sowie bösartige Neubildungen an Glottis, Zungengrund oder Kehlkopf. Von der
\end{abstract}

Analyse ausgeschlossen waren Patienten, bei denen ein flexibles Endoskop verwendet oder bei denen Kehlkopf oder Stimmlippen reseziert worden waren.

Innerhalb von sieben Tagen nach der Laryngoskopie hatten sich 232 Patienten (3,0\%) erneut beim behandelnden Arzt vorgestellt. Bei 21 (0,27\% der Gesamtkohorte) waren gravierende respiratorische Komplikationen der Anlass dafür, am häufigsten Stridor, Atemnot oder Atemversagen (8 Patienten) bzw. Larynxstenose oder Atemwegsödeme (6). Durch Sauerstoffmangel verursachte Hirnschäden wurden nicht beobachtet. Bei zwölf Patienten $(0,15 \%)$ traten andere schwere Komplikationen auf. Dabei handelte es sich um Synkopen oder Kollaps (4), Pneumonie (4), Sepsis (2) und Wheezing oder Schmerzen beim Atmen (2). Leichtere Komplikationen wurden bei $58 \mathrm{~Pa}$ tienten $(0,75 \%)$ erfasst. Dazu zählten u. a. Schmerzen, Dysphagie, Übelkeit und Dehydratation. In den sieben Tagen nach der Prozedur ereigneten sich außerdem zwei Todesfälle. $\mathrm{Zu}$ den genauen Umständen können den Studienautoren zufolge aufgrund der Schweigepflicht keine Angaben gemacht werden.

Fazit: Die HNO-Ärzte kommen zu dem Schluss, dass die direkte Laryngoskopie bei erwachsenen Patienten auch ambulant „ein günstiges Sicherheitsprofil“ aufweise. Voraussetzung sei jedoch ,ein vernünftiges Urteil“ bei der Auswahl der Patienten. „Nicht alle können unter ambulanten Bedingungen sicher behandelt werden, die präoperative Risikostratifizierung hat oberste Priorität." Patienten mit hohem Risiko für eine Blutung, Atemwegsobstruktion oder kardiopulmonale Komplikation sollten ebenso wie Patienten, die im Fall eines Problems nicht gleich einen Arzt aufsuchen können, bevorzugt stationär aufgenommen werden.

Dr. Beate Schumacher

Orosco RK et al. Safety of Adult Ambulatory Direct Laryngoscopy: Revisits and Complications. JAMA Otolaryngol Head Neck Surg 2015, online 2. Juli

\title{
Nasenballon hilft beim Paukenerguss
}

\section{Die konservative Behandlung eines Paukenergusses kann durch das Aufblasen spezieller Luftballons mit der Nase unterstützt werden. Der Nutzen der soge- nannten Autoinflation wurde jetzt auch in einer randomisierten Studie belegt.}

A n der Studie, die Ärzte um Ian Williamson, Universität Southampton, veröffentlicht haben, waren 320 Kinder im Alter zwischen vier und elf Jahren beteiligt. Alle hatten einen per Tympanogramm bestätigten Paukenerguss und waren zuvor durch einen Hörverlust oder andere typische Symptome aufgefallen. Kinder mit Ohrenschmerzen, Fieber oder anderen klinischen Zeichen einer akuten Otitis media waren ausgeschlossen.

Alle Studienteilnehmer erhielten die übliche Versorgung, die Hälfte wendete zusätzlich die Autoinflation an. Dazu sollten sie dreimal täglich einen speziellen Ballon mit der Nase aufblasen, das jeweils andere Nasenloch muss dabei mit dem Finger zugedrückt werden, der Mund geschlossen

\section{bleiben. Wenn nach einem Monat das} Tympanogramm immer noch für einen Erguss sprach (Typ B), sollte die Behandlung auf drei Monate verlängert werden.

Nach einem Monat war bei $47,3 \%$ der Kinder mit und bei 35,6\% ohne Autoinflation der Erguss verschwunden (Tympanogramm Typ A, C1 oder C2). Nach drei Monaten war dies bei $49,6 \%$ vs. 38,3\% der Fall. Das Aufblasen der Ballons hatte die Heilungschancen damit signifikant verbessert, nach Abgleich anderer Risikofaktoren um 36\% bzw. 37\%. Der Nutzen der Behandlung war unabhängig von Alter, Geschlecht und auch Schweregrad des Seromukotympanums.

Nasenbluten als mögliche Nebenwirkung der Behandlung war ähnlich häufig wie in der Kontrollgruppe (15\% vs. $14 \%$ ). Atemwegsinfekte (15\% vs. $10 \%)$ und Otalgien (8 vs. 2 Patienten) wurden in der Interventionsgruppe häufiger berichtet.

Fazit: Williamson und Kollegen schließen aus den Daten, dass die Autoinflation bei Kindern mit chronischem Tubenmittelohrkatarrh auch in der Primärversorgung machbar und wirksam ist. Weil der Erguss nicht immer vollständig verschwindet bzw. häufig rezidiviert, sei jedoch auch bei den behandelten Patienten weiterhin besondere Wachsamkeit angezeigt. Außerdem eigne sich die Autoinflation wahrscheinlich nicht für Kinder unter vier Jahren. Das Verfahren wird auch in der deutschen S1-Leitlinie zum Seromukotympanum als Behandlungsoption aufgeführt.

Dr. Beate Schumacher

Williamson I et al. Effect of nasal balloon autoinflation in children with otitis media with effusion in primary care: an open randomized controlled trial. CMAJ 2015, online 27. Juli 